\title{
Examining the Social and Cultural Barriers Present for Women Seeking Healthcare in Rural Communities of Karachi, Pakistan Nofel Karatela', Maria Altaf', Shamoon Noushad ${ }^{2}$, Amna Khan ${ }^{2}$, Shershah Syed ${ }^{4} \&$ Sadaf Ahmed $^{2,}$
}

1. University of Florida

2. Advance Educational Institute and Research Centre

3. Department of Physiology, University of Karachi

4. Koohi Goth Hospital

\begin{abstract}
Study was an interview-based cross-sectional survey with an objective of investigating if there are any social or cultural factors present in rural areas, which makes it difficult for women living in these communities to seek proper health care services. The subjects of this study were rural women who came to receive treatment for their gestation and related complications at Koohi Goth Hospital in Karachi, Pakistan. The variables that were explored included the level of education, socioeconomic status, social/family support, and level of awareness regarding proper birth care. Data collection included interviewing these women who were admitted to the outpatient department (O.P.D.) of the hospital and asking them questions concerning these variables. Much of the participants who were included for the study were conceiving for the 3rd and 4th time and they were in 3rd week of gestational period. The result of this study showed that women living in the rural areas were less educated due to their low socio-economic status. Disproportionate number of women claimed that learning about maternal health and related issues were never included in education they received.
\end{abstract}

Keywords

health care services, gestation, rural communities, awareness

\section{Introduction}

The rural communities of Pakistan and much of the countries with similar socioeconomic status including the Indian subcontinent encounters the major problem as the disproportionate lack of opportunity for health care. It has continuously afflicted certain groups of people to the point where it serves as a major source of health disparities. Another common trend of these communities seems to be the lack of knowledge regarding their health. Members of the community become complacent with practices they have been doing for years and are not taught to question these practices consequently leading to unnecessary health complications. Although there have been attempts to spread the awareness in rural communities, there seems to be a shortcoming of maintaining this type of education amongst rural communities. Another important factor within the context of one of these rural communities has been observed that it is especially women who commonly seem to lack the necessary support needed in a variety of aspects of health care (Ensor, T., \& Cooper, S., 2004).

Therefore, there are two realities that are most important to integrate in order to view the issue from an accurate and wholesome perspective: gender-based barriers and the fact that much of the climate of these rural communities consists of low education and lower awareness level. With an issue as delicate as birth, it is imperative that all women seeking to give birth have all the necessary conditions that may promote a healthy birth for both the baby and the mother. But far too often do complications 
occur for them just due to lack of knowledge that they need to go to a healthcare facility. As explored in this study, the reasons for this tendency to not seek healthcare have been seen to be social and cultural barriers that prevent the woman from even being willing to seek healthcare. In areas where extreme gender-based barriers are commonly observed, it is a reality that immobility and minimal income support have deprived women of improving their health outcomes. Gender disparities have caused a variety of health consequences due to the tendency of early marriage for the women in the community. Also, the majority of these women have dismally low literacy levels and levels of awareness which too commonly results in early child bearing with minimal to no information about proper patterns of seeking healthcare. These factors have certainly culminated into causing a high incidence of disease within this community due to improper health practices (Azmat, S. K., et al., 2012).

A subset of effects due to these barriers have seen to be low literacy levels of mothers, large family size, inordinate use of home remedies without proper diagnosis of the problem. This study serves to prove evidence that these social barriers exist and are factors influencing access to healthcare. In this study, the objective was to investigate if there are any social or cultural factors present in rural areas that make it difficult for women living in these communities to seek proper health care services. The subjects of the study were rural women who came to receive treatment for their gestation and related complications at Koohi Goth Hospital in Karachi, Pakistan. The variables that were explored were level of education, awareness, and socioeconomic status. Data collection included interviewing these women who were admitted to the outpatient department
(O.P.D.) of the hospital and asking them questions concerning these variables.

There are many social and cultural trends in rural areas of Pakistan, Bangladesh, and India that affect the access or willingness to seek medical treatment for obstetric problems. These tendencies are significant to address because they can help to expose influential causes of the lack of access to necessary medical treatment and why women aren't readily seeking the treatment they need. There has been an array of research that explores cultural beliefs and social limitations and how they affect an access to medical healthcare barrier. Previously a study was conducted which, investigated certain cultural barriers that ensure a lack of knowledge of contraceptive and family planning practices. Although this study does not specifically address the access to medical care, it does demonstrate how cultural barriers are even prevalent for the onset of these frequent pregnancies in rural areas. It does not only affect access to medical care, but also the rate of pregnancy occurrence. This is significant and supports our study because it illustrates the dynamic effect that social and cultural barriers have on gestation complications (Shaikh, B. T., \& Hatcher, J., 2005).

Salam (2008) explored specific cultural beliefs that demote the value of medical treatment in the eyes of these rural women. Salam and her colleagues carried out a survey based study that examined 30 Muslim respondents' answers to a short survey. One significant result that they found was that $65 \%$ of respondents agreed that it was required for a spousal male to give permission in order to seek medical treatment at an institution. This represents that the male-centric aspects of these cultural beliefs have a significant impact on the women's propensity to go seek treatment. Also, this 
study offers another interesting explanation of a cultural belief that affects why the women do not seek care (Azmat, S. K., et al 2013). The researchers found that the primary concern that the women expressed in the survey is that the medical treatment would cause them to expose themselves too much. The cultural tendency of these very religious women to show modesty and wear "hijabs" causes them to not be willing to have a physician examine their complications because it would overexpose them. This fear is especially heightened because of how they assume that a male physician will be examining them, which would seem to them as a taboo. The specific effects of these cultural tendencies support our research and help to found its purpose as significant because all of the women interviewed were members of highly religion-based communities (Lichtenstein, B., 2003). Social support also plays a significant role on the propensity of women to visit healthcare centers. A study conducted by Daniels examines the effect of social support on the number of pregnancy complications a black woman experienced in rural areas of the United States. He found that low social support correlated with a higher number of complications and higher support correlated with less complication (Shaikh, B. T., \& Hatcher, J., 2005).

\section{Methodology}

cross-sectional survey was conducted in collaboration with Koohi Goth Women Hospital \& Advance Educational Institute and Research Center. The data collection commenced in June 2015 and was completed in September 2015. The data was collected via interviews for which the approval of Institution Ethics Committee was preobtained. The information was collected on the basis of an interviewer-administered based questionnaire. The study instrument was developed in English and then translated into local languages. Quality control for the survey included visits to the field by experts and duplicate examinations by field supervisors. In total, 234 subjects were enrolled in the study where the survey response rates were uniformly excellent. Results were analyzed by using SPSS version 21.0.

\section{Results}

The data indicates that all of the patients are belonging to low-income families with minimal education. Lack of social support was seen with some women but many claimed to have a functional support system. Many of the women lived very far away from any healthcare facility, spanning from a 30minute distance to 2 hours of a distance. (responses were given in terms of time it takes to arrive at the healthcare facility). Most importantly, almost every woman interviewed claimed that they had minimal to no knowledge about proper birth care. Many of the women had given birth many times, ranging from 1-8 times and subsequently, much of them were at-home based births. Many of the women reported needing permission from their spouse to seek the health care facility, which characterizes an important social barrier. These indicators will help provide useful evidence that there are important social and cultural variables that serve in posing a major hindrance for women to maintain access to necessary medical treatment. In order to better understand and define predictors in addition, it is recommended to determine the role of cultural set up, social status and gender specific characteristics in this population with respect to means and values that are keeping. 


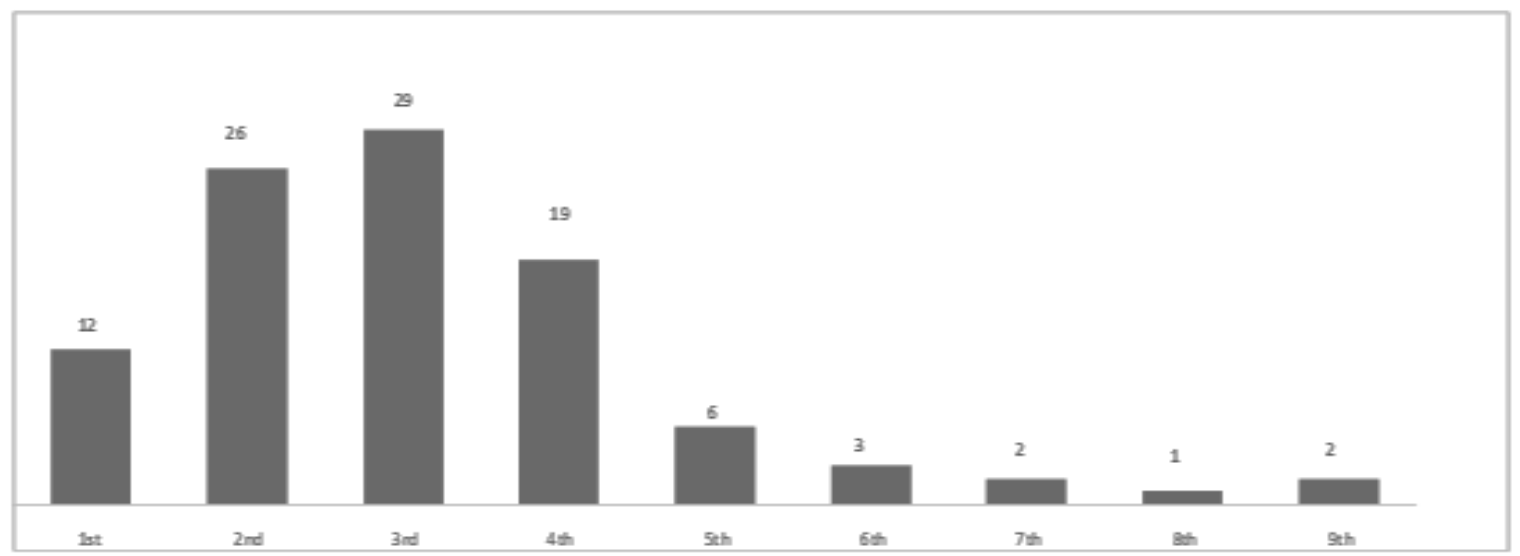

This figure shows the number of pregnancy for which the females visited hospital that was found to be with 3rd and 4th pregnancy on average. Though there were also many reported cases of missed pregnancies and abortions.

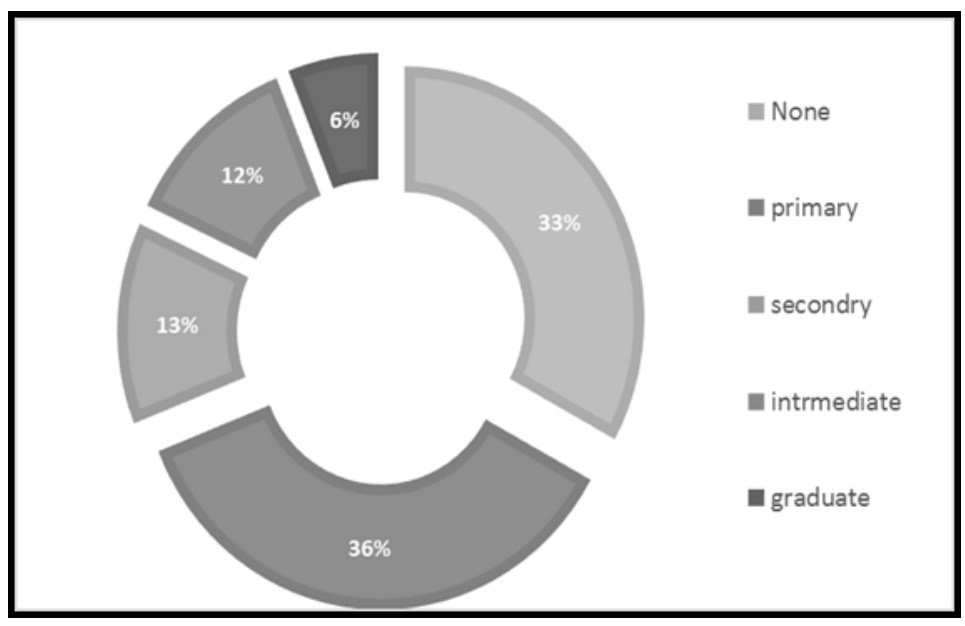

This figure shows the education level of subjects indicating that the low education status contributes to suppressed proper healthcare seeking behaviors that has a social background too.

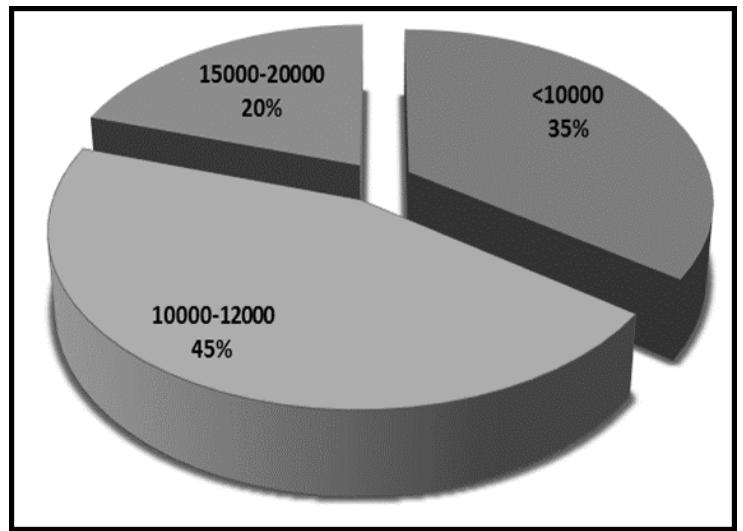

This figure shows the average income level of households of patient visiting hospital for basic health needs. Indicated that the families were economically weak to bear the cost of traveling and get easy access to facility. 


\section{Discussion}

There are some important factors to consider that may have had an influence on the responses that the women gave. One reality that is important to consider is that in Pakistani society, the role of the man in a family or community context plays an excessively powerful role in determining the future and fate of women seeking healthcare. This unfortunately propagates high levels of low self-esteem for the women causing them to abort their will of voicing their need and right to seek health attention. In such a situation, the woman may find herself burdened with this patriarchal reality, compounded with expectations that the community has for the woman (Shaikh, B. T., \& Hatcher, J., 2005). This study provides a basis for what further research should be founded by. It presents the variables and how women of the affected communities have expressed the variables that do affect their access to healthcare. Despite these seemingly insurmountable issues, there are certain ways that if maintained, they can serve to help alleviate the issues that many women in these rural areas face. First, there needs to be an active and collaborative effort to spread awareness and knowledge about proper birthcare (Khan, A., 1999). This may involve developing sustainable initiatives designed to hold community events that spread this knowledge. An important aspect of an initiative like this would be to attempt to directly involve leaders of the community. In doing such, the community is being empowered to take charge of their health care and propagate proper knowledge that might serve to tackle health complications that the community faces. To alter the mindset and improve the knowledge in a way that it permeates throughout the community is certainly a feat; but that does not mean an initiative should not be started (Mustafa, G., et al., 2015).

\section{Recommendations}

Basic healthcare awareness to men and women are alike in order to understand the gender roles better. Trained female personnel are required to attend to health issues of women in order to overcome the cultural norms. Educating people to better understand healthcare issues and utilize the available resources rising above the societal stigmas.

\section{Conflict of Interest}

We do not have any Conflict of interest.

\section{References}

- Azmat, S. K., Mustafa, G., Hameed, W., Ali, M., Ahmed, A., \& Bilgrami, M. (2012). Barriers and perceptions regarding different contraceptives and family planning practices amongst men and women of reproductive age in rural Pakistan: a qualitative study. Pakistan Journal of Public Health, 2(1), 17-23.

- Azmat, S. K., Shaikh, B. T., Hameed, W., Mustafa, G., Hussain, W., Asghar, J., ... \& Bilgrami, M. (2013). Impact of social franchising on contraceptive use when complemented by vouchers: a quasiexperimental study in rural Pakistan. PLoS One, 8(9), e74260.

- Ensor, T., \& Cooper, S. (2004). Overcoming barriers to health service access: influencing the demand side. Health policy and planning, 19(2), 69-79.

- Khan, A. (1999). Mobility of women and access to health and family planning services in Pakistan. Reproductive health matters, 7(14), 39-48.

- Lichtenstein, B. (2003). Stigma as a barrier to treatment of sexually transmitted infection in the American deep south: issues of race, gender and poverty. Social Science \& Medicine, 57(12), 2435-2445.

- Mustafa, G., Azmat, S. K., Hameed, W., Ali, S., Ishaque, M., Hussain, W., ... \& Munroe, E. (2015). Family planning 
knowledge, attitudes, and practices among married men and women in rural areas of Pakistan: Findings from a qualitative need assessment study. International journal of reproductive medicine, 2015.

- Salam, S. (2008). Cultural Barriers in Health Care Delivery to the Muslim Women of the USA. Health \& Medicine.
- Shaikh, B. T., \& Hatcher, J. (2005). Health seeking behaviour and health service utilization in Pakistan: challenging the policy makers. Journal of Public Health, 27(1), 49-54.

- Shaikh, B. T., \& Hatcher, J. (2005). Health seeking behaviour and health service utilization in Pakistan: challenging the policy makers. Jo 